This item was submitted to Loughborough's Research Repository by the author.

Items in Figshare are protected by copyright, with all rights reserved, unless otherwise indicated.

\title{
Investigating the potential to simulate hospital-based infection outbreaks using the STAMP architecture
}

PLEASE CITE THE PUBLISHED VERSION

http://conferences.theiet.org/system-safety/programme/index.cfm

PUBLISHER

(C) Institution of Engineering and Technology (IET)

VERSION

AM (Accepted Manuscript)

LICENCE

CC BY-NC-ND 4.0

REPOSITORY RECORD

Waterson, Patrick, and Paul Wai Hing Chung. 2019. "Investigating the Potential to Simulate Hospital-based Infection Outbreaks Using the STAMP Architecture”. figshare. https://hdl.handle.net/2134/7943. 
This item was submitted to Loughborough's Institutional Repository (https://dspace.lboro.ac.uk/) by the author and is made available under the following Creative Commons Licence conditions.

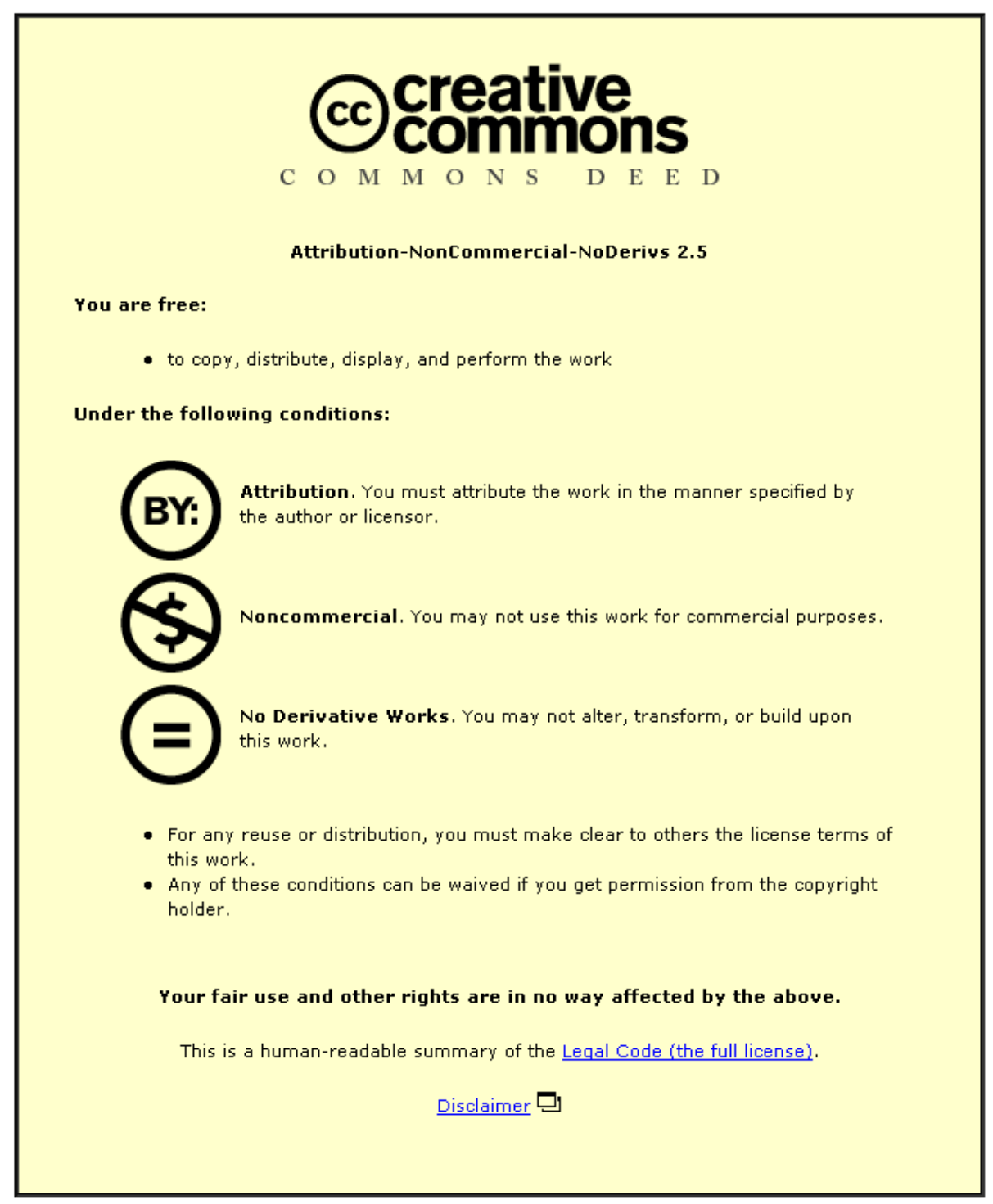

For the full text of this licence, please go to: http://creativecommons.org/licenses/by-nc-nd/2.5/ 


\title{
INVESTIGATING THE POTENTIAL TO SIMULATE HOSPITAL-BASED INFECTION OUTBREAKS USING THE STAMP ARCHITECTURE
}

\author{
P.E. Waterson ${ }^{*}$, P.W.H. Chung ${ }^{* *}$ \\ *Department of Ergonomics (Human Sciences), Loughborough University, UK, \\ *** Department of Computer Science, Loughborough University, UK \\ Email: p.waterson@lboro.ac.uk, p.w.h.chung@lboro.ac.uk
}

Keywords: STAMP; simulation and modeling; patient safety infectious diseases; complex systems.

\begin{abstract}
In this paper we describe our use of the STAMP architecture to analyse the $C$. difficile infection outbreaks which occurred at the Maidstone and Tunbridge Wells NHS trust. Background data from other outbreaks in the UK (e.g., at Stoke Mandeville NHS trust) were also used to build STAMP models. The specification uses components of STAMP such as negative and positive feedback loops to represent the degree to which decisions taken at a higher level in the system (e.g., bed turnover targets) impacted upon system components at lower levels (e.g., the morale and attitude of staff at ward level). In addition, the specification includes elements which aim to model the degree to which organisational learning was inhibited or facilitated within the system (e.g., through the provision of balancing loops Kontogiannis, in press). Finally, we examine the role played by the different types of mental model held by the various human actors in the system and how these change over time/are impacted by external constraints.
\end{abstract}

\section{Introduction}

The last few years have seen an increase in activity and enthusiasm in using ideas from systems thinking and systems dynamics to model aspects of healthcare systems (e.g., hospitals, disease pathways). Homer and Hirsch [9] for example, argue that systems dynamics facilitates the modelling of a range of risk management factors such as hospital resources, human behaviour and violations to patient safety. Much of this type of work has been carried out on public health issues (e.g., disease screening, workforce planning) and has primarily involved researchers with expertise in operational research (OR).

At the same time, researchers in human factors and ergonomics have attempted to use risk management models (e.g., Rasmussen, [16]; Leveson, [12]) to understand the interaction between components of complex sociotechnical systems, particularly where these systems degrade over time and accidents/disasters occur as a result (e.g., infection outbreaks - Vicente and Christoffersen, []).

In our paper we describe the steps we have so far taken to bring together the perspectives offered by systems dynamics modelling and human factors accident frameworks in order to better understand the tendency of some complex sociotechnical systems, particularly hospitals, to 'drift to failure' (Hollnagel et al., [8]). In the next section we briefly review current work on the application of simulation and modeling techniques to hospitalacquired infections.

\section{Simulation and modeling of hospital infections}

The subject of hospital infection control and prevention has recently become the subject of much media attention in the UK (e.g., BBC Panorama, [2]). A number of high profile hospital outbreaks within the UK involving bacterium such as Clostridium difficile (C..diff.) and MRSA (Methicillin-resistant Staphylococcus aureus) have raised infection control into a central priority for the UK NHS and other health care systems worldwide (Allegranzi et al., [1]). It is estimated that about $9 \%$ of patients in UK hospitals have a hospital-acquired infection. The costs of increased lengths of stay in hospital and treatment are thought to be around $£ 1000$ million a year (Noah, [15]).

One response to this problem has been to build mathematical models and use techniques from operational research to simulate infection outbreaks and design interventions for their prevention. Grundmann and Hellriegel [5] for example, provide a review of the mathematical models which have been developed over the years. Stochastic models have proved to be every common, these involve the use of data relating to frequency of hand washing and resource levels (e.g., number of isolation beds or staff time), setting parameter values for these and then using random variabilities built into the model to calculate the likelihood of infections spreading. An alternative approach, drawn from OR is to use agent-based models to simulate patients (e.g., colonization, treatment and location within the ward). Meng et al. [14] have used an agent-based architecture to suggest assess a range of policies for the treatment of MRSA (e.g., screening tests, decolonization treatment). 
Most modelling efforts have so far focused on using infectionrelated parameters as inputs, little work has examined the role played by behavioral factors, particularly those that cut across a range of levels of analysis (i.e., individual, group and organisational) and involve other actors outside of the immediate hospital environment (e.g., NHS regulators). In order to demonstrate the value and importance of this these types of considerations, we describe in section 3 the background to a set of recent outbreaks in the UK relating to the $C$. difficile bacterium.

\section{Recent hospital outbreaks of $C$. difficile in the UK}

C. diff. is the major cause of serious bacterial infectious diarrhoea acquired in hospitals in the UK and is particularly resistant to drying, chemical disinfectants and alcohol. The number of cases of C. difficile and reported deaths has increased within the UK in the last few years and similar outbreaks have occurred at a number of hospitals (e.g., Stoke Mandeville - Healthcare Commission, [7]). Recommendations for the prevention of the spread of the bacteria include: the timely isolation of known and suspected cases; the control of antibiotics; the application of high standards of hygiene; and, the restriction of the movements of patients.

The outbreaks of bacterium $C$. difficile occurred at the Maidstone and Tunbridge Well NHS Trust between 2005-2007 and resulted in the death of approximately 90 people. The Healthcare Commission's investigation report in 2007 [6] identified five main factors which contributed to the outbreaks: (1) Lack of adequate coordination between external organisations and regulators; (2) The style of management and leadership within the hospital trust; (3) Poor clinical management and decision-making on the hospital wards; (4) Management of the infection control team; and, (5) Inadequate levels or hygiene and run-down buildings/equipment.

Based upon these findings, Waterson [21] used Rasmussen's [16] risk management framework to analyse potential whole system and cross-level relationships within the report findings (figure 1). For example, one of factors that appears to have shaped decisionmaking across the various levels of the system (e.g., regulatory, trust governance, clinical management levels) is likely to have been financial and bed occupancy targets set by other actors within the system (e.g., the Strategic Health Authority acting on behalf of the Government). Decision-making within the system as a whole showed characteristics of what Weick and Sutcliffe [22] found in their analysis of the Bristol Royal Infirmary report, in that staff became locked in patterns of behaviour without challenging the likely adverse impact these would have on patients.

Based upon the outcomes form this work we decided to use some of the elements from Leveson's [12] STAMP methodology to further explore the dynamic inter-relationships that exist between factors leading up over time to the outbreaks and system components. STAMP was chosen partly because it uses elements of the risk management framework and provides a means of considering the role dynamics multi-level relationships of in accident causation.

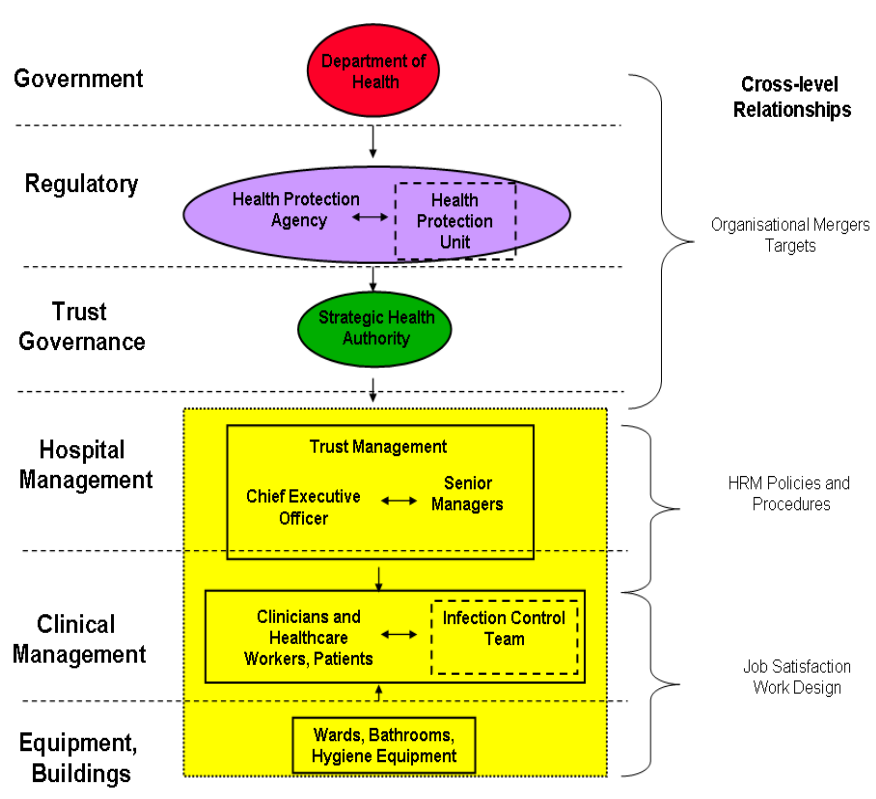

Figure 1: Cross-level relationships in the Maidstone and Tunbridge Wells Outbreak [21]

\section{Using STAMP to simulate the outbreaks}

\subsection{The STAMP architecture}

From the various papers written about STAMP (e.g., [11], [12]) it is difficult precisely identify the main components of the architecture. In order to facilitate analysis we have used the description given by Leveson in her analysis of the Walkerton outbreak of $e$. Coli in Canada [12]. This version of STAMP consists of the following components: (1) a model of the organisational safety structure control structure; (2) a model of the dynamics and pressure that lead to system degradation over time; (3) process models that required to control and maintain the system (including feedback and communication requirements).

\subsection{Steps in using STAMP}

Our first step in using STAMP was to consolidate the findings from two of the recent outbreaks in the UK (Maidstone and Stoke Mandeville, [6], [7]). This involved looking at the commonalities between the outbreaks and then organizing these into causal maps. An example drawn from the Maidstone outbreak is shown in figure 2. This, along with outline data from the Stoke Mandeville outbreak, was used as the basis for the three steps followed by Leveson in her use of STAMP with the Walkerton outbreak (sections 4.4.1-4.2.3) 


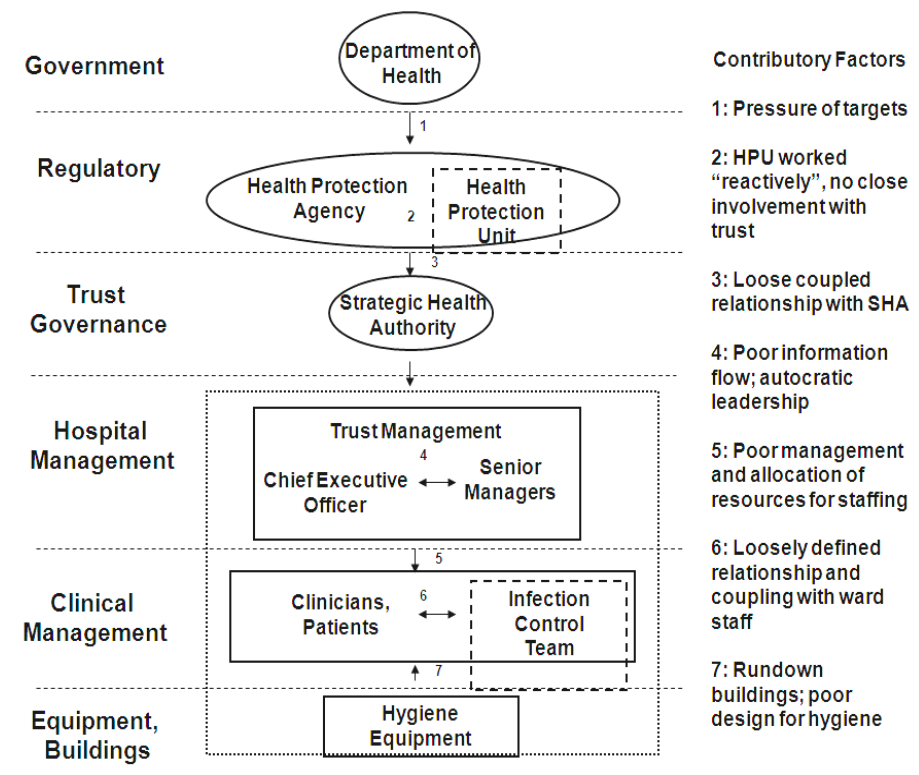

Figure 2: Causal mapping between contributory factors in the Maidstone and Tunbridge Wells outbreak [21]

\subsubsection{Safety control structure}

Figure 3 shows an example of the safety control structure which was constructed on the basis of the two infection outbreaks.

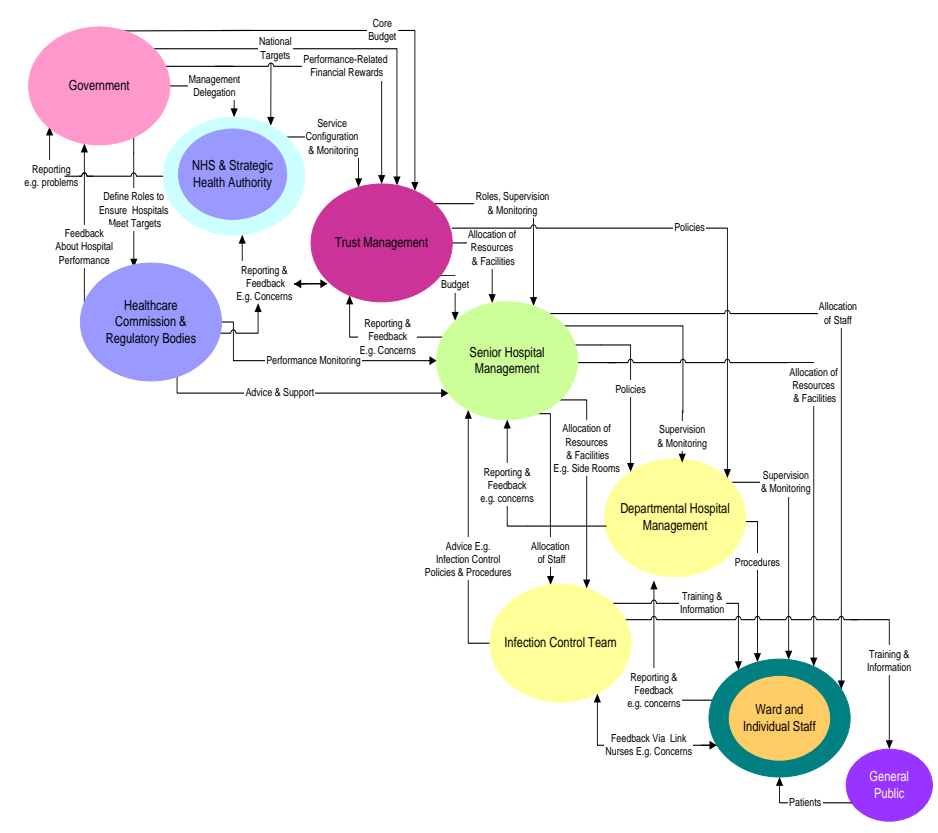

Figure 3: STAMP safety control structure based on [6], [7], [21].

The safety control structure is an attempt to identify the main system hazards, safety constraints and the overall control structure which enforces these. Each component within the overall control structure has a set of safety constraints associated with it. For example, the infection control teams in both outbreaks were responsible for providing training and information to ward staff, in addition to carrying other functions such as advising and feedback regarding infection policy/procedure to senior managers within the hospital.

\subsubsection{Changes over time leading up to the outbreaks}

Identifying exactly the nature of system degradation or 'drift to failure' within the two outbreaks involves some conjecture and inference in order to fill in missing information. The reports on both outbreaks provide some detail of the various contributory factors leading up to the outbreaks, but accounts of how this changed over time are limited. Nevertheless, it is possible, based on accounts of other health care accidents as well as the general context of changes within the NHS, to suggest how the various aspects of the safety control structure changed over time.

At the very highest ('macro') level of the system government targets placed many individuals, particularly those at trust board and management levels under a great deal of pressure. Within the trusts it is likely that targets exerted considerable pressure on the system as a whole and this pressure filtered down various levels of the system. The drive to comply with these targets increased the likelihood of an adverse event or set of events taking place at some stage within the trust.

The creation of the Health Protection Agency (HPA) in April 2005 coincided with the first outbreak at the Maidstone and Tunbridge Wells trust. The remit of the HPA is to provide advice and support to NHS, local authorities and other agencies with regard to public health issues. One part of the HPA, the health protection unit (HPU), is to support organisations in their management of infections. The investigation report [] highlighted the confusion this caused within the trust at the time of the outbreaks as the expectation was that the HPU could give provide guidance covering the supervision and monitoring of infection control. Similar problems were encountered within the much larger Strategic Health Authority (SHA), the report mentions that until recently the SHA were more focused on outbreaks of other healthcare associated infections (e.g., MRSA), partly because these were at the time of the outbreaks one of the national priority to which performance targets were attached. One possibility in the case of the HPA and other regulatory bodies is that over time regulatory enforcement procedures, particularly with regard to $C$. difficile took on some of the characteristics of other management failings within other parts of the system (e.g., confusion of responsibilities). This type of so-called 'agency capture' as been shown to occur in other descriptions of accidents and disasters and the changes which occur over time (e.g., []).

The style of leadership within the trusts and the overall management culture were also problematic. Within Maidstone staff described the leadership of the chief executive as being "autocratic" or "dictatorial". In addition, the person appointed as director of infection prevention and control had "no real understanding of the role at the outset" $[6, p .5]$. Turnover of managers and directors was high. Between September 2002 and September 2006 five people attended the board in five roles as director or director of finance [6, p. 91]. Despite weekly meetings 
there was little evidence of managers working collaboratively to address problem. Managers were often under considerable stress and in fear of losing their jobs [6, p. 92]. Likewise most staff were afraid to speak out and raise concerns about what was happening within the trust.

Finally at lower ('micro') levels of the system, the behaviour of clinicians and other health care professionals within the trust shares similarities with those of senior managers and trust board managers. Many individuals at ward level were aware of the levels of poor hygiene and inadequate patient monitoring practices, but saw no way to improve the situation. Other researchers have suggested that in such cases individuals fall into the trap of routinely carrying out their work without challenging assumptions or questioning the consequences of what they are doing ("involuntary automaticity" - [18]). In such cases organisational learning throughout the system is inhibited and the ability to adapt and learn from failure results in patterns of negative reinforcement which, in turn, act as a barrier to change and improvement [22].

\subsubsection{Dynamic process model}

The third step in using the STAMP methodology involves using the static description of the control structure together with changes to this structure over time, to build a dynamic control structure. The purpose of this is to identify why the system changed over time [11, p. 191] and uses systems dynamics to cary out modeling of feedback loops and time delays. Figure 4 shows one part of the dynamic process model for the Maidstone and Tunbridge Wells outbreak.

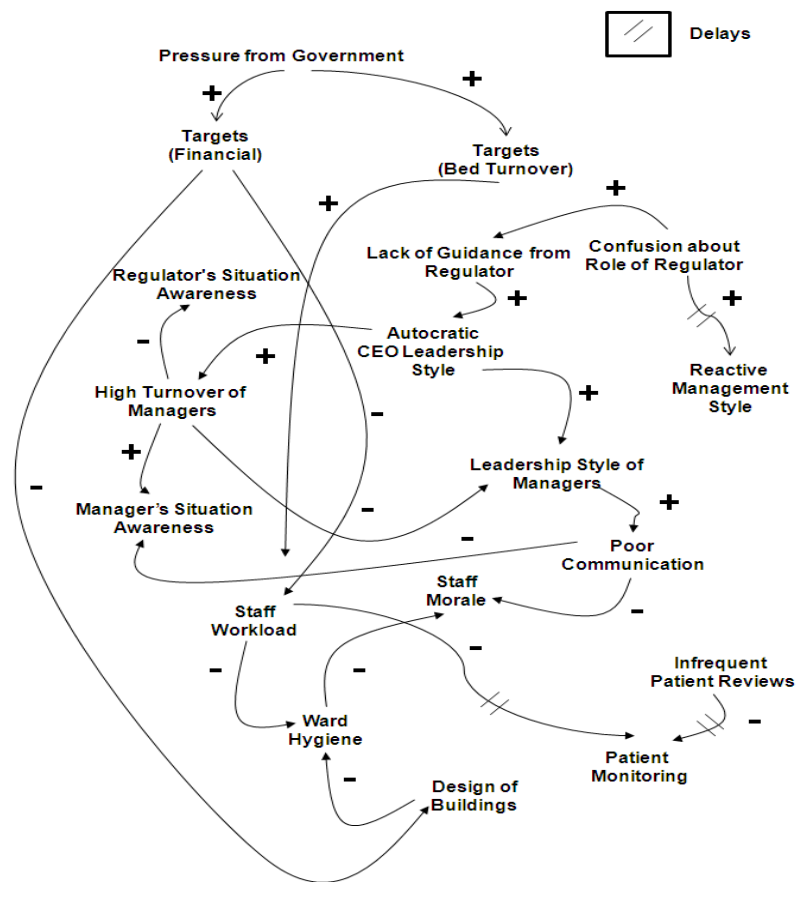

Figure 4: Example dynamic process model of infection outbreak

\section{Discussion}

Our use of the STAMP architecture follows on from a systems analysis using Rasmussen's Risk Management (ActorMap) Framework [16]. In general we found that following the three steps leading up to the construction of a dynamic process model help to provide further insights into the outbreaks. These went beyond our earlier analysis [21] in that they helped to highlight a number of aspects of the outbreaks that warrant further investigation.

Our earlier work had focused on the role of cross-level and whole system relationships in causing the outbreaks (figures 1 and 2). By contrast, the construction of the control structure and dynamic process model provided another way of looking at the issue of how the various trusts drifted into eventual failure. Putting together the elements in figure 4 for example, helped to operationalise the tendency of all of the actors within the systems to become complacent over time about the level of risk associated with the $C$. difficile infection. The gap between the situation awareness of senior managers within the trusts and ward staff is hinted at within the various investigation reports, but is not explicitly identified as a cause of the outbreaks. Lack of a shared mental model of the risk of infection across regulatory, management and staff system levels combined this 'drift to complacency', was brought out by using STAMP. In particular, the use of a systems dynamics format for the dynamic process model, helped to focus our attention on some of the 'latent' causes of the outbreaks. Similar types of advantage have been described by other analyses using systems dynamics (e.g., Cooke's analysis of the Westray mine disaster - [3]).

Our use of STAMP also pointed to some potential disadvantages of the architecture. Firstly, we found it difficult to identify exactly the core stages of analysis and what the exact procedure should be in carrying a STAMP analysis. Secondly, it proved to be easier to build the control structure to represent the main hazards and constraints within the trust, as compared to the dynamic process model. The latter involved a degree of arbitrariness and ambiguity in assigning attributes to the process model (figure 4). This may have been a side effect of the lack of detail regarding the actions/decisions of specific individuals and groups in the investigation reports. However, it seems that along with the identification of specific attributes, the assignment of positive/negative feedback loops involves a degree of subjectivity on the part of the analyst. As a result issues regarding the reliability of the methods (i.e., whether or not two or more analysts will arrive at similar conclusions) remain open and require further investigation.

\section{Future work}

The work we have described is in a very early stage. The various models which were derived from using STAMP need to be refined and developed further. In particular, the dynamic process model (figure 4) needs to be expanded and to take into account more detailed findings from other infection outbreak case studies. Part of the analysis pointed to the issue of the drift to 
complacency which seems to have been characteristic of both outbreaks. One potential future avenue of research is to focus on this more specifically. Marais et al. [13] for example, outline a set of archetypes for organisational safety alongside descriptions of associated process models for each. One of these archetypes concerns organisational complacency and how this phenomenon arises over time. The description of the process model could be used to further describe how complacency and apathy in the face of growing infection risk contributed to the outbreaks. In addition, the taxonomy of variability of organisational processes described by Kontogiannis [10] could be used to examine further aspects of the outbreaks in ore depth.

Our current work involves an attempt to build a small-scale computational simulation of components within the dynamic process model described above. STAMP has served as a basis for carrying out preliminary systems analysis and the precise choice of implementation (e.g., agent-based modeling, system dynamics environments) is yet to be made. These types of simulation are becoming more common, particularly within health care [17] and other domains where there is a need to further explore causal links between macro- and micro-system elements [23].

\section{Acknowledgements}

We would like to thank Helen Cheetham for her work on the Stoke Mandeville outbreak which contributed toward the STAMP analysis.

\section{References}

[1] B. Allegranzi, J. Storr, G. Dziekan, A. Leotsakos, L. Donaldson and D. Pittet. "The first global patient safety challenge "Clean Care is Safer Care" from launch to current progress and achievements". Journal of Hospital Infection, 65 (supplement 2): 15. (2007).

[2] BBC Panorama (2008), "How Safe is Your Hospital?" (Broadcast on $27^{\text {th }}$ April, 2008), (available at: http://www.bbc.co.uk/mediaselector/check/player/nol/newsi d_7370000/newsid_7371100? redirect=7371116.stm\&news $=$ $1 \&$ nbram $=1 \&$ nbwm $=1 \&$ bbram $=1 \&$ bbwm $=1 \% 20 \quad$ (Last accessed $28^{\text {th }}$ April, 2008)

[3] D.L. Cooke. "A systems dynamics analysis of the Westray mine disaster”. Systems Dynamics Review, 19, 139-166. (2003)

[4] W. Freundenberg. "Bureaucratic slippage and the failures of agency vigilance: The case of the environmental studies program". Social Problems, 41, 501-26 (1994)

[5] H. Grundmann and B. Hellriegel, "Mathematical modeling: a tool for hospital infection control", Lancet Infectious Diseases, 6, 39-45. (2006)

[6] Healthcare Commission. "Investigation into outbreaks of Clostridium difficile at Maidstone and Tunbridge Wells NHS Trust",

(http://www.healthcarecommission.org.uk/_db/_documents/ Maidstone_and_Tunbridge_Wells_investigation_report_Oct _2007.pdf. (2008).
[7] Healthcare Commission "Learning from Investigations" (http://www.healthcarecommission.org.uk/_db/_documents/ Learning_from_investigations.pdf (2008).

[8] E. Hollnagel, D.D. Woods and N.G. Leveson. Resilience Engineering. Aldershort: Ashgate. (2006).

[9] J.B. Homer, J.B. and G.B. Hirsch, G.B. "System dynamics modelling for public health: background and opportunities". American Journal of Public Health, 96, 3, 452-458. (2006)

[10] T. Kontogiannis. "A contemporary view of organizational safety: variability and interactions of organizational processes". Cognition, Technology and Work. (2010, in press)

[11] N.G. Leveson. M. Daouk, N. Dulac and K. Marais. "Applying STAMP in accident analysis" Paper presented at the Workshop on Investigation and Reporting of Incidents and Accidents (IRIA), September. Available at: http://shemesh.larc.nasa.gov/iria03/p13-leveson.pdf (2003)

[12] N.G. Leveson. "A new accident model". Safety Science, 42, 237-270. (2004)

[13] K. Marais, J.H. Saleh and N.G. Leveson. "Archetypes for organisational safety”. Safety Science, 44, 565-582. (2006)

[14] Y. Meng, R. Davies, K. Hardy and P. Hawkey. "An application of agent-based simulation to the management of hospital-acquired infection". Journal of Simulation, 4, 6067. (2010).

[15] N. Noah, "Controlling Communicable Disease". Maidenhead: Open University Press. (2006).

[16] J. Rasmussen. "Risk management in a dynamic society: a modeling problem". Safety Science, 27, 183-213. (1997),

[17] L. Termine et al. "NOSOSIM: an agent-based model of pathogen circulation in a hospital ward". Proceedings of the 2009 Spring Simulation Multiconference. San Diego, California. (2009).

[18] B. Toft and H. Mascie-Taylor. "Involuntary automaticity: a work-system induced risk to safe health care". Health Services Management, 18, 211-216. (2003)

[19] A. Tucker and A. Edmondson. "Why hospitals don't learn from mistakes: organizational and psychological dynamics that inhibit system change". California Management Review, 45, 1-18. (2003)

[20] K.J. Vicente and K. Christoffersen. The Walkerton E. Coli outbreak: a test of Rasmussen's framework for risk management in a dynamic society. Theoretical Issues in Ergonomics Science, 7, 2, 93-112. (2006)

[21] P.E. Waterson "A systems ergonomics analysis of the Maidstone and Tunbridge Wells infection outbreaks". Ergonomics, 52, 1196-1205. (2009)

[22] K.E. Weick. and K.M. Sutcliffe. "Hospitals as cultures of re-enactment: a re-analysis of the Bristol Royal Infirmary". California Management Review, 45, 73-84. (2003)

[23] G.L. Zacharias, J. MacMillan and S.B Van Hemel (Eds.). "Behavioural Modeling and Simulation" Washington: National Academies Press. (2008). 